\title{
Towards a BIM-agile method in architectural design Assessment of a pedagogical experiment
}

\author{
Henri-Jean Gless ${ }^{1,2}$ [0000-0001-7676-2632], Gilles Halin ${ }^{1,2}$, and Damien Hanser ${ }^{1}$ \\ ${ }^{1}$ MAP-CRAI (UMR n 3495), École Nationale Supérieure d’Architecture de Nancy \\ 2 rue Bastien Lepage, 54000 Nancy, France \\ ${ }^{2}$ Université de Lorraine, 54000 Nancy, France \\ gless@crai.archi.fr
}

\begin{abstract}
This paper describes a scientific experiment carried out in the context of the AEC in France. This research is part of the digital transition in architecture, with a particular interest in BIM technology and how to integrate it into architectural design through social sciences.

Indeed, the arrival of BIM technology raises both technical and human questions. The design work is changed, the amount of work is moved upstream, but above all we see new tools, new uses, and new practices without any project management method emerging. In other fields such as industry, software engineering and HMI design, we have seen the emergence of methods that focus more on the team and the user than on the process. We find Lean, continuous improvement, or agility, a family of methods that interests us here.

Our research hypothesis is that inserting agile practices alongside current business practices will integrate and exploit BIM technology and other digital innovations.

To do this, we identified what the problems were with BIM technology, and selected several agile practices highlighting communication, group cohesion and customer needs identification to address them. Thus, we carry out experiments in which we test, analyze and adapt these agile practices to architectural design.

This paper then describes a pedagogical experiment conducted with Master 2 students at the École Nationale Supérieure d'Architecture of Nancy in France. In a workshop, the students had to carry out a BIM project, while they used the agile practices that we had adapted: the design matrix, the micro poker, and the standup meeting. In addition to these three practices, we took the opportunity to try agile overseeing using what we call a stand-up meeting. The objective is to validate the synergy of these practices while ensuring that they respond to our communication, group cohesion and customer needs integration issues.

This experiment takes place over one week and will serve as a basis for us to prepare experiments in a professional context.
\end{abstract}

Keywords: Architectural design, Agile methods, Agile practices, BIM Technology, Collaborative design, Intentions elicitation, Lean management, Project management, Social sciences. 


\section{Introduction}

This research takes place in the field of AEC industry in France where the digital adoption is underway with BIM technology still not properly implemented. Nowadays, for both the educational and the professional field this transition is often seen only through the technological prism and not through the social science approach.

As part of our research, we are developing so-called agile methods and collaborative practices to facilitate the adoption and the exploitation of BIM in architectural design. Agility is a term derived from the world of software engineering, and consists of human-centered methods and practices. In order to validate our hypotheses before testing them in the professional world, this paper describes an experiment with architecture students. Moreover, our hypothesis is that training students in new digital practices is an efficient way for changes to take place in the professional world.

During a workshop week called Design and Digital Manufacturing, the students put in groups must design a BIM architectural project before making a scale model 1/20 using 3D printers and laser cutters. They will apply three agile practices that we have adapted for architectural design: micro poker, design matrix and stand-up meeting. We have tested these practices individually. We want to show that they can improve communication in a collaborative working group, have a better group cohesion, and have a better integration of client needs. This experiment will allow to verify if these practices are compatible together. In order to ensure that the experiment is going well, we take the opportunity to insert another practice: the supervision of a BIM-agile coach. He ensures that the workshop runs smoothly, and deals with problems concerning the other practices.

We will focus in part 2 on the current context of digital transition in France. Next, we will see in part 3 how BIM technology disrupts work habits, and what locks are identified. Part 4 will focus on the practices proposed to address these blockages. Finally, Part 5 will concern the experimentation of these practices with our students.

\section{A sector in transition without changes in management}

While the digital transition is taking place in the AEC industry, other sectors are adapting and creating innovative project management methods.

\subsection{France at the heart of digital change}

The world of architecture, engineering and construction (AEC) in France, particularly in the field of architectural design, is currently going through an important period of change; digital practices and collaborative practices are changing and adapting while BIM technology is becoming both a regulatory requirement in public construction and a productivity requirement in complex projects. However, there is a certain inertia on the part of architects towards Building Information Modeling. This trend can be explained by the size of French architectural firms. Most of them are small (90\% have 9 
employees or fewer and 75\% have 4 employees or fewer) [1]. Also because of the current socio-economic context, which favors a low level of investment in the medium and long term. Since each agency has its own method of doing architecture, they have already become involved in project management, and the arrival of a tool like BIM raises questions [2].

\subsection{Emerging project management practices}

In the field of project management, methods are emerging from continuous improvement or Lean Production, as Lean Construction [3]. In order to improve the construction, it is necessary to ask the question of the place of the coordination in the whole process. According to Patrick MacLeamy's curve, BIM technology increases the amount of work upstream [4] and changes digital and collaborative practices, which can generate misunderstandings. We have identified communication, group cohesion, and customer needs satisfaction problems with the use of BIM technology. Results in a climate of mistrust between the actors of a project, and rigidifies coordination. In software engineering and HCI design fields, agile methods are being applied to answer similar issues [5].

\section{$3 \quad$ BIM technology needs agile practices}

The digital transition brings new technologies such as BIM, but for the latter, no framework is precisely described. There is a need for new management methods.

\subsection{BIM technology brings complexity}

BIM is defined as a technology, which designates both a process and a design tool. However, it is actually a digital model in which designers will insert more or less information depending on the progress and complexity of the project. BIM stands for Building Information Model, Modeling, or Management. However, it only brings tools and uses (Model and Modeling) and no human coordination practices (Management) [6], which does not necessarily allow good exploitation, and therefore limits its integration with architects and agencies.

These new tools and uses change the way to do project management. The amount of design and modeling work is indeed shifted upstream of the design phase. By passing from a 2D design to a 3D (and more) design, the amount of work is also increased or moved upstream. This is also the case when we add semantic and enriched notions [7]. It becomes mandatory to think in more detail about object modeling.

By bringing more work and more complexity upstream, BIM technology brings more tasks and more decision-making processes and forces designers to bring more coordination earlier. These changes are transforming the collaborative process, with more information to be shared earlier among stakeholders. BIM poses the problem of the complexity but also creates a need for exchanges between the stakeholders. 


\subsection{A need for elicitation, refinement and evaluation}

We conducted interviews with students and architectural design professionals in order to confirm our hypothesis about the exchanges. Our interlocutors are generally informed and aware of the BIM concept but remain concerned about its arrival. Moreover, we found that collective activities concerning BIM tasks are difficult activities, and more particularly their definition, their "owners", their transmission to other actors (with their initial meaning), or their estimated duration. We call that elicitation, refinement and evaluation activities [8].

Thus, we have oriented our research towards practices to improve collaboration and consequently exchanges within a group in order to solve these problems: the agile methods.

\subsection{Agility as a coordination vector}

Agile methods are specific management methods that involve the customer in the decision process and follow three other fundamental rules: team collaboration, continuous improvement and change acceptance [9].

Our hypothesis is that the insertion of agile practices into design activities (therefore the four basic rules will improve communication, group cohesion and customer integration) will improve the quality of the architectural project. Indeed, agile methods and practices focus on building trust between all the design actors (the customer including).

\section{$4 \quad$ Agile practices identification and adaptation}

We have thus looked at the agile practices employed in the world of software engineering and the HCI design, and identified four of them. Three are practices used by the stakeholders themselves and one is a coaching practice. We experimented and adapted them to the architectural design.

\subsection{Design matrix: writing down intentions}

The design matrix is an online table that is filled out collaboratively. Inspired by Suh's matrices [10], we have created it to allow the actors of the collaboration to confront their architectural intentions. The programmatic elements of the project, referred to as the inputs, are found in rows; the needs or deliverables, referred to as outputs, are listed in columns.

This practice allows the actors to elicit collaboratively their intentions, and to ask themselves all the questions they have in mind about the project: the concept, the references, the keywords, etc.

Moreover, this practice provides a reflective framework for design, a concrete basis to help the less creative or comfortable actors with a pencil to still give their ideas. 


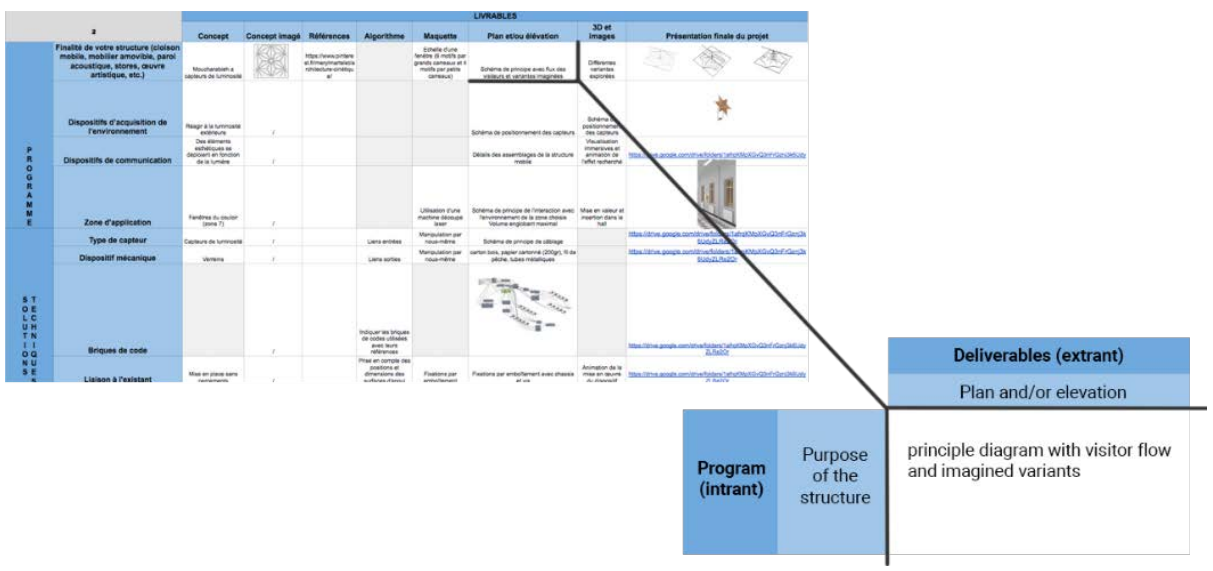

Fig. 1. Zoom on how to fill a design matrix with text (could be schemas, pictures or URL).

\subsection{Micro poker: put everybody on the same page}

Micro poker is an agile practice that we have adapted from planning poker [8, 11]. It is a deck of cards, and each player has four cards. There are several scales of values such as numbers (1, 20, 80 and 100) or color (green, yellow, orange or red) or size (S, M, L and $\mathrm{XL}$ ). In order to properly identify a design task, players ask questions, and all of them answering at the same time by choosing a card.

Once everyone has played, the players with the furthest cards begin to debate their choice. Once everyone has spoken, the group must converge towards a compromise. Then start another round.

This practice allows players to express themselves and elicit their thoughts, while avoiding the phenomenon of the "first speaker". This phenomenon is the fact that, during a debate, the protagonists set their arguments against those of the person who spoke first.
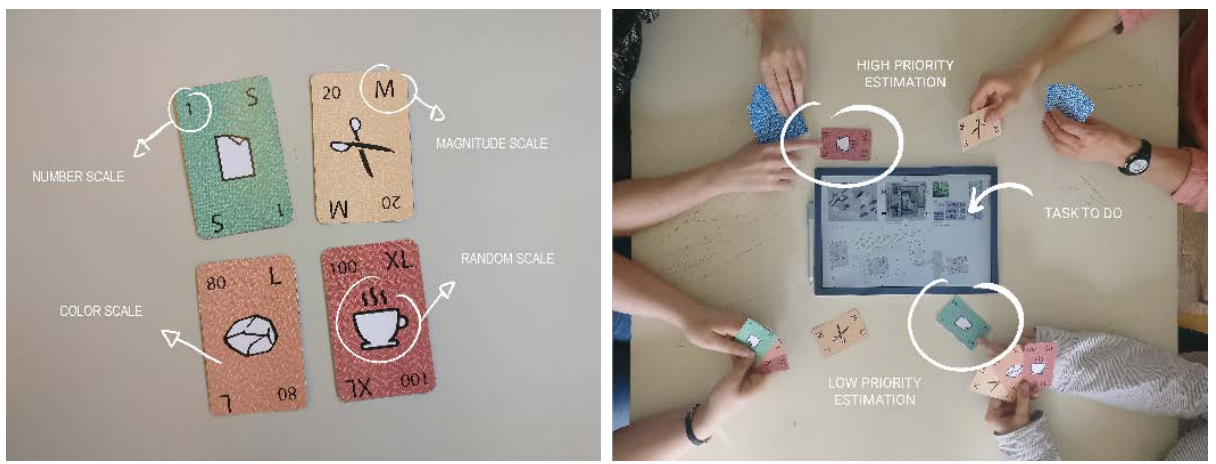

Fig. 2. A set of Micro Poker cards (left) and four players during a round (right). 
This is an example of an exchange sequence:

Actor 1: How long does it take to place the point cloud in the 3D model we scanned yesterday? We use S, M, L or XL. (everybody chooses a card, and reveals it at the same time; actor 1 and actor 2 with extreme card start talking).

- Actor 1: I have put a S card because it is not difficult to import a cloud point in the software.

- Actor 2: I do not agree! I have put a XL card because by experience, there is always something that goes wrong, and we need to clean the point cloud before import it.

- Actor 1: OK I have not thought about that.

- Actor 3: And we need to finish the ground modeling before that.

- Actor 2: Right! OK, I will clean the cloud, during this time, can you finish the ground?

- Actor 1: I will help him, I am comfortable with that. So, for the next round: how long does it take to finish the ground? (Here starts a new round). [8]

\subsection{Stand-up meeting: taking stock}

The stand-up meeting is an agile practice that consists of making a short daily meeting among the actors of the collaboration [11]. These actors stand and talk about what they did yesterday, what they are going to do today, and what do they need if they have problems.

This practice allows to know what other actors do and to develop cohesion and a shared knowledge of the project. People remain standing in order to stay focused away from their workstations, and also to minimize the comfort and therefore the duration of the meeting. They want to get back to work.

The goal is to have a quick global vision of the progress and the problems encountered. An actor who is above the project often leads this practice in order to bring objectivity and help. This practice did not need much adaptation: a graphic document seems, however, relevant in an architectural design context. Indeed, it allows to have what is called deliverables, kind of design milestones reorienting the work and thus to integrate the customer's needs.
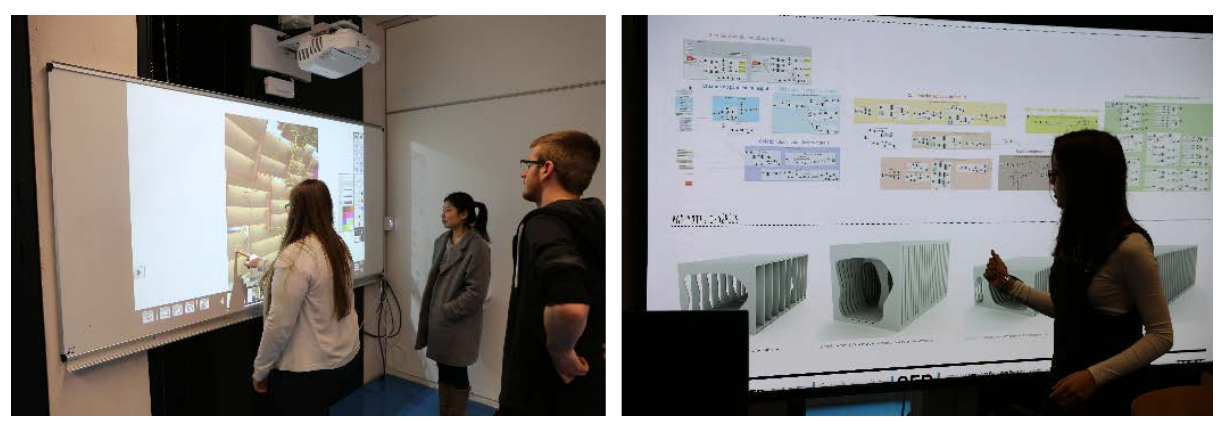

Fig. 3. Examples of two Stand-up Meetings with graphic supports. An engineer showing her assembling (left); an architect showing variants of its algorithm (right). 


\subsection{A BIM-agile coach to oversee the workshop}

Experimentation here consists of knowing if they are compatible and beneficial to the use of BIM. The students have already experienced the different practices independently of each other but can still ask themselves some questions. One of the practices we had previously identified that did not require students' participation is the BIM-agile coach or coordination facilitator. This person knows perfectly the three other practices, as well as the project that the students must carry out, and supervises them in order to answer their questions about the project, technical issues or coordination.

This practice comes from the scrum method [11] where we find a scrum master and a product owner. The first one is in charge of the well application of the method, and the second one represents the customer needs and views about the project. In our pedagogical context, we adapted them both in order to merge into a BIM-agile coach.

\section{The four practices in experimentation}

In order to validate their semester, the 20 students from the Master $2 \mathrm{AME}^{1}$ must make a workshop called Design and Digital Manufacturing. They can apply everything they have learned during the year, about BIM, project management and design.

\subsection{Design and Digital Manufacturing workshop}

Students experimented in February during the Design and Digital Manufacturing workshop our agile practices. The students' work topic consisted of the realization of an architectural object of their choice, related to mobile micro architecture. Students came from different curricula, so each group contained at least an architect, an engineer and a designer. The topic and the composition of the groups were sent to them beforehand so that they could appropriate it during the weekend. They already have been introduced to agility with a course, and with some exercises.

We distributed a set of micro poker to each student, and they could use it when they want as a decision-making tool throughout the week, as well the design matrix. The stand-up meeting was held every morning. The agile coach was there during the whole week to provide support.

In order to measure the benefits of these agile practices, we were interested in the students' use of our tools or simply whether they were using them during the whole week; the students also received at the end of the week a survey so that they can give their opinion on agility.

\subsection{A protocol for one semester}

Before conducting the experiment, we gave courses and exercises about agility and project management to our students.

1 AME (Architecture, Modeling and Environment) is a Master 2 course from the École Nationale Supérieure d'Architecture (National School of Architecture) of Nancy, France. 
We followed this protocol:

- Theoretical phase: opening course on agility and project management followed by an explanation of the three agile practices;

- Practical phase: one exercise per practice;

- Practical phase: a semester long BIM collaborative project studio;

- Data gathering: students do a report about the previous collaboration exercise, with comments on practices;

- Practical phase: the Design and Digital Manufacturing workshop;

- Data gathering: recordings and observations during the DDM and anonymous online survey about agility after it;

- Data analysis: bias sort and practices improvement.

The theoretical phase allows students to understand project management by studying its history and to realize that there are important changes when adopting digital technologies. It is also a phase allowing an introduction to agile methods and practices.

The first practical phases consist of exercises where we present our agile practices. The second practical phase is a collaborative BIM project where they are free to apply the practices they wish. Finally, they end the semester with the MDD workshop where we ask them to implement our practices.

The first data gathering phase mainly consists to organize an oral presentation and to offer students agile feedback; this prepares the DDM workshop week. The second one is doing recordings and distributing an anonymous survey that gives us qualitative feedback.

Finally, the data analysis phase consists of a sorting of the data previously collected, in order to eliminate misunderstanding biases of the subject, or irrelevant answers of the survey.

\subsection{Observations during the workshop}

The BIM-agile coach could observe how the students used the practices to experiment in addition to his support work. We made the following observations:

- Design matrix: it is used at the beginning of the workshop to allow students to write and draw the ideas they have. On the other hand, it is used less and less as the week progresses.

- Micro poker: students tend to use poker not as an elicitation and refinement tool, but as a voting system.

- Stand-up meeting: students were reluctant to use it at the beginning, thinking they didn't need it. On the $2^{\text {nd }}$ day, they asked for it in order to take stock and "officially" make their choices.

- BIM-agile coach: this made it possible to quickly solve the problems of timetable, interpretation of the subject and techniques.

We realized that tools can be used less as time goes on. This is normal: no more need for decision support tools when the project is advanced and only production tasks remain to be carried out. 


\subsection{The survey results}

Two weeks after the intensive workshop, we distributed a survey to students to gather their opinions on the practices used and agility in general. The results are rather positive in the sense that out of 20 people, there is never less than $60 \%$ satisfaction and never more than $15 \%$ bad criticism on agility.

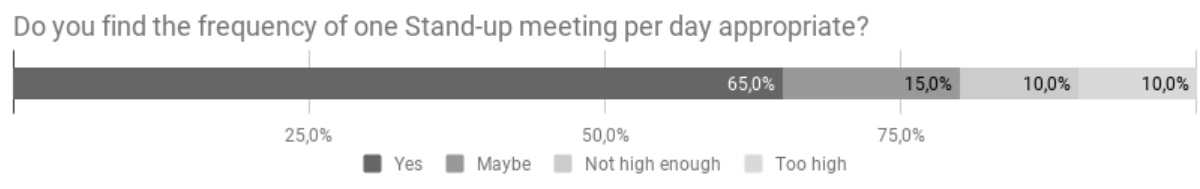

Do you think that the Stand-up meeting allowed you to be more organized?

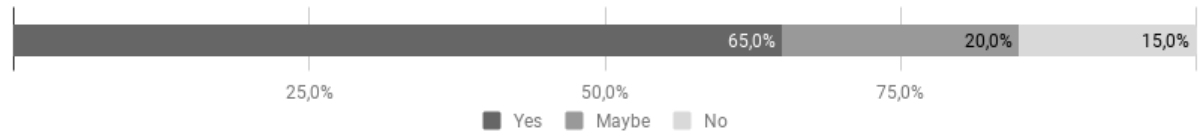

Do you think agility was useful for your project?

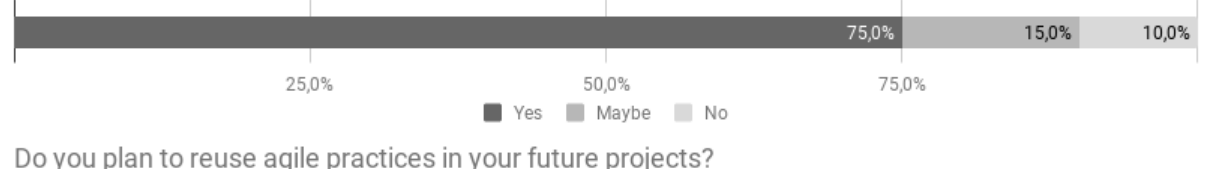

Do you plan to reuse agile practices in your future projects?

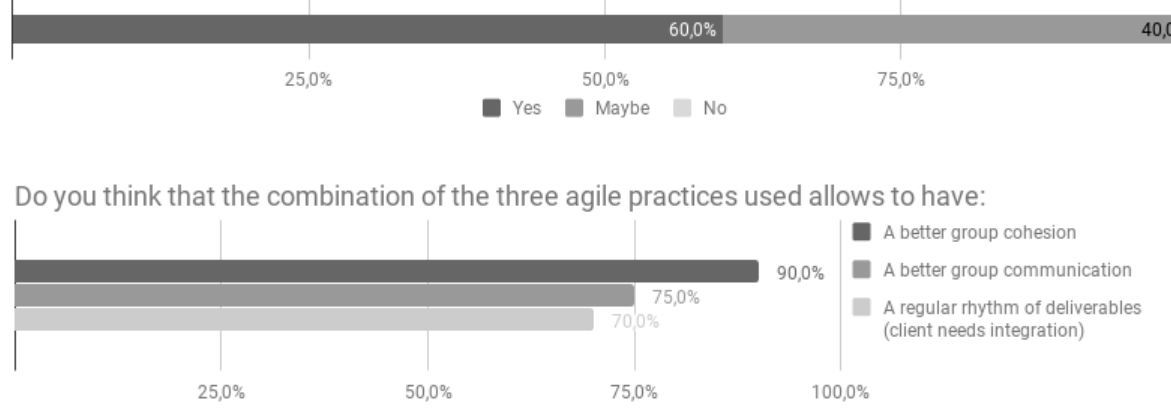

Fig. 4. Some results of the student survey conducted after the workshop.

More specifically, 65\% of students found the frequency of one stand-up meeting per day appropriate, while $10 \%$ found it too low and $10 \%$ too high. The same proportion of students are satisfied with the practice's contribution to their organization. A large proportion, $75 \%$ of the 20 students, thinks that agility was useful in carrying out their project. However, only $60 \%$ or 15 points less plan to reuse agile practices in their future projects. One can qualify with the fact that no student ever plans not to reuse them.

Finally, a majority of students found that the three agile practices they used allowed them to have a better group cohesion, a better communication, as well as a rhythm of deliverables allowing them to stick to the needs of the statement (a virtual customer in a certain way). 


\section{Conclusion and opening on professional experiments}

The observations made during the workshop and the results of the survey allow us to say that the agile practices we have proposed have been beneficial for collaboration.

First of all, all three student practices were used, and the survey shows their willingness to apply agile practices again. The design matrix was not completed to the end, but as said, the tool was no longer pertinent after a while. Micro poker allowed students to make decisions quickly, and they even made it their own. For the record, they sometimes used it to decide what to eat. The stand-up meeting was the practice that really made it possible to complete such a complex project in such a short time. Having a regular cycle of deliverables and being able to take stock every day allowed them not to get lost in projects too ambitious or too far from the subject. Finally, the BIM-agile coach served on both sides: an interlocutor for them who solves problems and a "pressure" sensor for us.

To conclude, this experimentation has confirmed that agility can play a major role in the integration of BIM and new technologies into architectural design. We will then try our agile practices in a professional environment in order to continue our research.

\section{References}

1. Observatoire de la profession d'architectes, Archigraphie, p. 48. https://www.architectes.org/sites/default/files/atoms/files/archigraphie-coul-2016-web_0.pdf, last accessed 2018/04/02 (2016).

2. Hochscheid, E. and al.: BIM Implementation in SMEs: an Experience of Cooperation between an Architect Agency and a Carpentry Firm. International Conference on Computing in Civil and Building Engineering, 6-8 July 2016, Osaka, Japan (2016).

3. Dupin, P.: Le LEAN appliqué à la construction : Comment optimiser la gestion de projet et réduire coûts et délais dans le bâtiment ? $1^{\text {rst }}$ edn. Eyrolles, Paris (2014).

4. Kensek, K. and al.: Manuel BIM : Théories et Applications. Eyrolles (2015).

5. Womack, J., Jones, D.: Lean Thinking: Banish Waste and Create Wealth in Your Corporation, Revised and Updated. $2^{\text {nd }}$ edn. Free Press, New-York (2003).

6. Zignale, D. and al.: Modelling practices and usages to improve adaptation of groupwaretool services: Application in the AEC sector. EuropIA.13: 13th International Conference on Advances in Design Sciences and Technology. Roma, Italy (2011).

7. Halin, G., Gallas, M.: Une approche pédagogique par les modèles pour la sensibilisation au concept de BIM (Maquette Numérique) SCAN'16, 7-9 September 2016, Toulouse, France (2016).

8. Gless, H.-J. and al.: BIM-agile practices experiments in architectural design. In: Luo Y. (eds) Cooperative Design, Visualization, and Engineering. CDVE 2017. Lecture Notes in Computer Science, vol 10451, pp. 135-142. Springer, Cham (2017).

9. Beck, K. et al.: Manifesto for agile software development. http://agilemanifesto.org (2001)

10. Suh, N.: Axiomatic Design: Advances and Applications. Oxford University Press, Oxford (2001).

11. Kniberg, H.: Scrum and Xp from the Trenches: How We Do Scrum. $2^{\text {nd }}$ edn. Lulu.com (2015). 